\title{
Bidirectional Connectivity between Hemispheres Occurs at Multiple Levels in Language Processing But Depends on Sex
}

\author{
Tali Bitan, ${ }^{1}$ Adi Lifshitz, ${ }^{2}$ Zvia Breznitz, ${ }^{2}$ and James R. Booth ${ }^{3}$ \\ ${ }^{1}$ Department of Communication Sciences and Disorders and ${ }^{2}$ Department of Learning Disabilities, Edmond J. Safra Brain Research Center for the Study of \\ Learning Disabilities, University of Haifa, Haifa 31905, Israel and ${ }^{3}$ Department of Communication Sciences and Disorders, Northwestern University, \\ Evanston, Illinois 60208
}

Our aim was to determine the direction of interhemispheric communication in a phonological task in regions involved in different levels of processing. Effective connectivity analysis was conducted on functional magnetic resonance imaging data from 39 children (ages $9-15$ years) performing rhyming judgment on spoken words. The results show interaction between hemispheres at multiple levels. First, there is unidirectional transfer of information from right to left at the sensory level of primary auditory cortex. Second, bidirectional connections between superior temporal gyri (STGs) suggest a reciprocal cooperation between hemispheres at the level of phonological and prosodic processing. Third, a direct connection from right STG to left inferior frontal gyrus suggest that information processed in the right STG is integrated into the final stages of phonological segmentation required for the rhyming decision. Intrahemispheric connectivity from primary auditory cortex to STG was stronger in the left compared to the right hemisphere. These results support a model of cooperation between hemispheres, with asymmetric interhemispheric and intrahemispheric connectivity consistent with the left hemisphere specialization for phonological processing. Finally, we found greater interhemispheric connectivity in girls compared to boys, consistent with the hypothesis of a more bilateral representation of language in females than males. However, interhemispheric communication was associated with slow performance and low verbal intelligent quotient within girls. We suggest that females may have the potential for greater interhemispheric cooperation, which may be an advantage in certain tasks. However, in other tasks too much communication between hemispheres may interfere with task performance.

\section{Introduction}

The role of interhemispheric communication is often thought of in the context of hemispheric lateralization of function and primarily left hemisphere specialization for language. Behavioral experiments using lateralized presentation of verbal stimuli (dichotic listening or tachistoscopic presentation to one visual field) found an advantage for stimuli presented to the left hemisphere (Kimura, 1961). One view explained this advantage in reduced efficiency in processing verbal stimuli in the right hemisphere, assuming independent processing in each hemisphere and no interactions between them (Iacoboni and Zaidel, 1996; Bogen, 2000). Another account has been that information presented to the nondominant hemisphere has to be transferred for processing in the dominant hemisphere, resulting in a delayed response (Nowicka et al., 1996; Hugdahl et al., 1997). The critical role of "callosal transfer" is evident from the suppression of verbal stimuli presented to the nondominant hemisphere in split brain patients (Musiek et al., 1989; Mohr et al., 1994; Sugishita et al., 1995) and from the negative correlation found between callosal volume and left lateralization of language (Galaburda et al., 1990;

\footnotetext{
Received March 10, 2010; revised June 16, 2010; accepted June 20, 2010.

This research was supported by National Institute of Child Health and Human Development Grant HD042049 to J.R.B.

Correspondence should be addressed to Tali Bitan, Department of Communication Sciences and Disorders, University of Haifa, Mt. Carmel, Haifa 31905, Israel. E-mail: tbitan@research.haifa.ac.il.

DOI:10.1523/JNEUROSCI.1245-10.2010

Copyright $\odot 2010$ the authors $\quad 0270-6474 / 10 / 3011576-10 \$ 15.00 / 0$
}

Westerhausen et al., 2006). Although there may be competition and inhibition between hemispheres (Kinsbourne, 1982; Bloom and Hynd, 2005), there is ample evidence for hemispheric cooperation (Gazzaniga, 2000) in electrophysiological (Endrass et al., 2002) and computational modeling studies (Weems and Reggia, 2004). While these studies suggest that verbal information is transferred between hemispheres, they do not indicate the specific level of processing at which information is transferred and whether the communication between hemispheres is unidirectional or reciprocal.

Recent functional neuroimaging studies showing that hemispheric specialization is a relative and dynamic phenomenon that depends on specific task parameters (Grimshaw et al., 2003; Jansen et al., 2006; Petit et al., 2007; Britton et al., 2009) imply that hemispheric communication may be reciprocal. This may involve both a signal from the specialized to the nonspecialized hemisphere and transfer of information from the nonspecialized to the specialized hemisphere with increasing task demands (Hellige, 1990; Banich, 1998; Weissman et al., 2000). Current methods of effective connectivity analysis in neuroimaging enable the examination of directional interhemispheric connectivity separately in regions involved in distinct levels of processing. Taken together, results from two effective connectivity studies using visual spatial and letter judgment tasks (McIntosh et al., 1994; Stephan et al., 2007) suggest that the main direction of influence from the nonspecialized to the specialized hemisphere or vice versa and the level of processing at which interhemi- 
Table 1. Participants age and IQ

\begin{tabular}{lllc}
\hline & Girls & Boys & Total \\
\hline Number & 22 & 17 & 39 \\
Age mean & $11: 10$ & $12: 4$ & $11: 9$ \\
Age range & $8: 8-14: 6$ & $8: 9-14: 4$ & \\
VIQ mean & 114.3 & 116 & 115 \\
VIQ range & $91-137$ & $79-142$ & 108 \\
PIQ mean & 107.5 & 108.9 & \\
PIQ range & $79-139$ & $78-134$ & \\
\hline
\end{tabular}

PIQ, Performance IQ; VIQ, verbal IQ.

spheric interactions occur may depend on the task and on the side the stimuli are presented.

Our goal was to examine interhemispheric interactions in children by using a phonological task with bilateral auditory presentation. While bilateral presentation is more natural, phonological processing is a strongly lateralized task (Scott et al., 2000). Moreover, findings showing a developmental increase in language lateralization (Brauer et al., 2008; Ressel et al., 2008) suggest that interhemispheric connectivity may become more asymmetric with age. To address questions of directionality and levels of processing, we used effective connectivity analysis between regions associated with sensory, phonological, and cognitive processing.

\section{Materials and Methods}

The data analyzed in this study were previously published in a study that focused on developmental changes in activation (Cone et al., 2008), and was also one of four tasks reported in a study that showed sex differences in activation (Burman et al., 2008). The current study comprises the first effective connectivity analysis on these data and focuses on hemispheric interactions.

\section{Participants}

Thirty-nine healthy children (17 boys) ages $9-15$ years participated in the study (see Table 1 for details). All children were native English speakers and right handed with normal hearing and normal or corrected-to-normal vision. All children were free of neurological disease or psychiatric disorders and were not taking medication affecting the CNS. None of the children had a history of deficits in intelligence, reading, or oral language, and none had a learning disability or attention deficit hyperactivity disorder. All participants were administered the performance and verbal portions of the Wechsler abbreviated scale of intelligence (Wechsler, 1999) with two verbal subtests (vocabulary, similarity) and two performance subtests (block design, matrix reasoning). Participants' standard scores are presented in Table 1. There was no significant difference between boys and girls in age $\left(t_{(37)}=0.65\right)$ or verbal intelligence quotient $(\mathrm{VIQ})\left(t_{(37)}=0.36\right)$ and no correlation between age and VIQ $(r=-0.26$, $p=0.11)$.

\section{Stimuli}

Rhyming task. Two English words were presented aurally in a sequential order and the participant had to determine whether the words rhymed and indicate their judgment by pressing one of two buttons. The duration of each word was between 500 and $800 \mathrm{~ms}$ followed by a brief period of silence, with the second word beginning $1000 \mathrm{~ms}$ after the onset of the first. A red fixation cross appeared on the screen after the second word, indicating the need to make a response during the subsequent $2600 \mathrm{~ms}$ interval. Twenty-four word pairs were presented in each one of four lexical conditions that independently manipulated the orthographic and phonological similarity between words. In the two nonconflicting conditions, the two words were either similar in both orthography $(\mathrm{O})$ and phonology $(\mathrm{O})\left(\mathrm{O}^{+} \mathrm{P}^{+}\right.$; e.g., dime-lime), or different in both orthography and phonology $\left(\mathrm{O}^{-} \mathrm{P}^{-}\right.$; e.g., staff-gain). In the two conflicting conditions, the two words had either similar orthography but different phonology $\left(\mathrm{O}^{+} \mathrm{P}^{-}\right.$; e.g., pint-mint $)$or different orthography but similar phonology $\left(\mathrm{O}^{-} \mathrm{P}^{+}\right.$; e.g. jazz-has $)$. If the words rhymed the participant pressed a button with the index finger, and if they did not rhyme the participant pressed a different button with the middle finger.

Control conditions. There were three kinds of control tasks. The simple perceptual control had 24 pairs of single pure tones, ranging from 325 to $875 \mathrm{~Hz}$. The tones were $600 \mathrm{~ms}$ in duration and contained a $100 \mathrm{~ms}$ linear fade in and a $100 \mathrm{~ms}$ linear fade out. The complex perceptual control had 24 pairs of three-tone stimuli, where all the component tones were within the aforementioned frequency range. Each tone was $200 \mathrm{~ms}$, with a $50 \mathrm{~ms}$ fade in and out. For both the simple and complex perceptual controls, participants determined whether the stimuli were identical or not by pressing a yes or no button. The tones were equal in maximum amplitude to the words, and the procedures for presenting stimuli were the same as those for the rhyme judgment task. The third control task involved 72 null events. The participant was instructed to press a button when a black fixation cross at the center of the visual field turned red. The fixation event had exactly the same visual stimuli and response characteristics as the rhyming task and the perceptual controls.

Stimulus characteristics. All words for the rhyme decision task were recorded in a soundproof booth using a digital recorder and a high quality stereo microphone. A female native English speaker read each word in isolation so that there would be no contextual effects. All words longer than $800 \mathrm{~ms}$ were shortened to this duration $(<1 \%$ of the words). All words were then normalized so that they were of equal amplitude. All words were monosyllabic and were matched across conditions for written word frequency in adults and children [using The Educator's Word Frequency Guide (Zeno et al., 1996)] and for adult word frequency for written and spoken language (Baayen et al., 1995).

\section{Experimental procedure}

After informed consent was obtained and the standardized intelligence test was administered, participants were invited for a practice session in which they were trained in minimizing head movement in front of a computer screen using an infrared tracking device. In addition, they performed one run of the rhyming task in a simulator scanner to make sure they understood the tasks and to acclimatize themselves to the scanner environment. Different stimuli were used in the practice and scanning sessions. Scanning took place within a week after the practice session. The rhyming task was administered in two 108 trial runs ( 8 min each) in which the order of lexical, perceptual, and fixation trials was optimized for event-related design (Burock et al., 1998) using OptSeq (http://surfer.nmr.mgh.harvard.edu/optseq/). The order of stimuli was fixed for all subjects.

\section{Magnetic resonance imaging data acquisition}

All images were acquired using a 1.5 T GE (General Electric) scanner. A susceptibility weighted single-shot echo planar imaging (EPI) method with blood oxygenation level-dependent contrast was used, and functional images were interleaved from bottom to top in a whole brain EPI acquisition. The following scan parameters were used: echo time (TE) = $35 \mathrm{~ms}$, flip angle $=90^{\circ}$, matrix size $=64 \times 64$, field of view $=24 \mathrm{~cm}$, slice thickness $=5 \mathrm{~mm}$, number of slices $=24$, and repetition time $(\mathrm{TR})=$ $2000 \mathrm{~ms}$. Each functional run had 240 repetitions. In addition, a high resolution, T1-weighted, three-dimensional image was acquired (spoiled gradient-recalled acquisition in a steady state, $\mathrm{TR}=21 \mathrm{~ms}, \mathrm{TE}=8 \mathrm{~ms}$, flip angle $=20^{\circ}$, matrix size $=256 \times 256$, field of view $=22 \mathrm{~cm}$, slice thickness $=1 \mathrm{~mm}$, number of slices $=124$ ), using an identical orientation as the functional images.

\section{Image analysis}

Conventional data analysis was performed using statistical parametric mapping (SPM5) (http://www.fil.ion.ucl.ac.uk/spm). The images were spatially realigned to the first volume to correct for head movements. No individual runs had $>4 \mathrm{~mm}$ maximum displacement, with an average of $1.2 \mathrm{~mm}$ per individual run. Sinc interpolation was used to minimize timing errors between slices (Henson et al., 1999). The functional images were coregistered with the anatomical image and normalized to the standard T1 template volume (Montreal Neurological Institute). The data were then smoothed with a $10 \mathrm{~mm}$ isotropic Gaussian kernel. Statistical analyses at the first level were calculated using an event-related design, with four lexical conditions of the rhyming task, two conditions of the 
Table 2. Regions of activation in all lexical conditions versus fixation at the group level at $\boldsymbol{p}<\mathbf{0 . 0 5}$ corrected for familywise error

\begin{tabular}{|c|c|c|c|c|c|c|c|}
\hline Region & $B A$ & $\mathrm{H}$ & Zscore & Voxels & $x$ & $Y$ & $Z$ \\
\hline \multirow[t]{3}{*}{ Superior temporal gyrus } & 22 & Right & $\operatorname{lnf}$ & 1162 & 60 & -27 & 6 \\
\hline & 22 & Right & $\operatorname{lnf}$ & & 51 & -15 & 6 \\
\hline & 22 & Right & Inf & & 63 & -9 & 3 \\
\hline \multirow[t]{3}{*}{ Superior temporal gyrus } & 22 & Left & $\operatorname{lnf}$ & 2188 & -45 & -21 & 6 \\
\hline & 22 & Left & $\operatorname{lnf}$ & & -63 & -9 & 0 \\
\hline & 22 & Left & Inf & & -60 & -33 & 6 \\
\hline Medial frontal gyrus & 8 & & 6.97 & 252 & -3 & 15 & 48 \\
\hline Lingual gyrus and middle occipital gyrus & 18 & Right & 6.01 & 922 & 24 & -57 & 0 \\
\hline Fusiform gyrus & 37 & Left & 5.75 & 61 & -45 & -54 & -18 \\
\hline Right inferior frontal gyrus & 47 & Right & 5.53 & 62 & 36 & 24 & -3 \\
\hline Parahippocampal gyrus & 38 & Left & 5.08 & 15 & -39 & 0 & -27 \\
\hline Middle frontal gyrus & 6 & Left & 5.07 & 20 & -30 & -9 & 63 \\
\hline Post central gyrus & 3,2 & Left & 4.7 & 11 & -45 & -33 & 60 \\
\hline
\end{tabular}

VOIs for the STG were selected from the local maxima to avoid overlap with the VOI of the primary auditory cortex A1. Coordinates of left IFG are not presented because this area was continuous with the left STG. The coordinates for the left IFG VOI [ -48 12 24] were identified using an anatomical mask of left IFG. Regions in bold were used as VOIs for the effective connectivity analysis. Inf, Inferior.

perceptual control, and the fixation events as seven conditions of interest. A high-pass filter with a cutoff period of $128 \mathrm{~s}$ was applied. Group results were obtained using random effects analyses by combining subject-specific summary statistics across the group as implemented in SPM5 (Penny et al., 2004). Active regions, in the contrast of "all lexical conditions versus fixation" at a threshold of familywise error corrected $p<0.05$, served as the group reference for the selection of the volumes of interest (VOIs) at the individual level.

\section{Effective connectivity analysis}

Six VOIs were chosen for the effective connectivity analysis. In the left hemisphere, the regions were inferior frontal gyrus (IFG), fusiform gyrus (FG), and superior temporal gyrus (STG), because these were previously identified as part of the language network involved in phonological judgment (Binder et al., 1994; Booth et al., 2004; Bitan et al., 2007), and the primary auditory cortex (A1) as the locus of direct sensory stimulation. In the right hemisphere, the regions included A1 and STG. Right IFG was not included in the model because activation was found only in pars orbitalis and not in regions homologous to the activation in left IFG, making questions of reciprocality irrelevant. Regional responses were summarized as the principal eigenvariates of responses within a $6 \mathrm{~mm}$ radius sphere. For the IFG, FG, and bilateral STG, the VOI was centered on the voxel with the strongest signal in the individual's activation map in the contrast of all lexicals versus fixation within $10 \mathrm{~mm}$ of the group peak activation and restricted by an anatomical mask of the relevant region defined based on WFU PickAtlas in SPM5 (Maldjian et al., 2003) (i.e., IFG: pars opercularis and pars triangularis; STG: superior and middle temporal gyri; and FG: fusiform and inferior temporal gyri). These relatively large anatomical masks were used to account for individual variability in the location of the maximally activated voxel. Fifteen participants had the peak IFG activation in pars triangularis, whereas 24 had the peak in pars opercularis. Furthermore, the peak activation that defined the center of the left STG VOI was on the border of the middle temporal gyrus in four participants. However, as can be seen in the supplemental text, available at www.jneurosci.org, as supplemental material, this variability did not affect the results of the model comparisons. For bilateral A1, the center of VOI was fixed across individuals and based on the group maximal activation in the contrast of "perceptuals versus fixation" within an anatomical mask of Heschl gyrus. Fixed coordinates for A1 were used to avoid overlap between A1 and STG VOIs and because all participants had above threshold activation in this location. Table 2 and Figure 1 show the results of the conventional analysis and the group reference for the VOIs. $z=6$

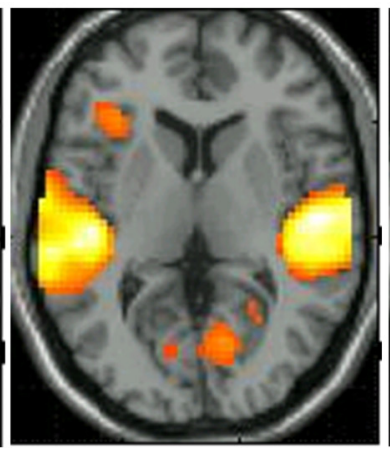

$z=24$

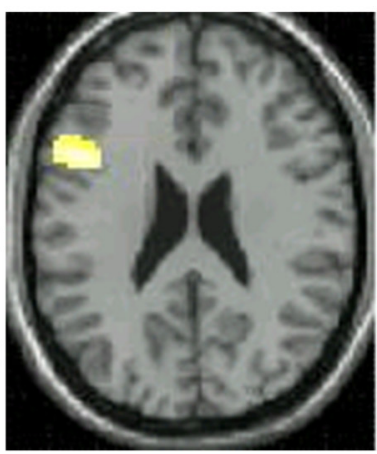

\section{6} 6 3 48 . 
Table 3. Strength of Intrinsic connections in the selected model (No. 10)

\begin{tabular}{lrrrrrr}
\hline & \multicolumn{7}{l}{ From } & & & \\
\cline { 2 - 6 } To & Right A1 & Left A1 & Right STG & Left STG & Left IFG & Left FG \\
\hline Right A1 & & -0.012 & $-\mathbf{0 . 0 3 7}$ & $\mathbf{- 0 . 0 3 7}$ & & \\
Left A1 & $\mathbf{- 0 . 0 4 4}$ & & $-\mathbf{0 . 0 3 9}$ & $-\mathbf{0 . 0 2 0}$ & & \\
Right STG & $\mathbf{0 . 2 5 1}$ & $\mathbf{0 . 2 8 7}$ & & $\mathbf{0 . 1 9 6}$ & $\mathbf{0 . 0 5 9}$ & \\
Left STG & $\mathbf{0 . 1 9 7}$ & $\mathbf{0 . 2 3 0}$ & $\mathbf{0 . 2 0 0}$ & & $\mathbf{0 . 0 7 3}$ & $\mathbf{0 . 0 4 5}$ \\
Left IFG & & & $\mathbf{0 . 1 6 3}$ & $\mathbf{0 . 1 7 8}$ & & $\mathbf{0 . 0 4 7}$ \\
Left FG & & & & $\mathbf{0 . 1 5 6}$ & $\mathbf{0 . 0 8 9}$ & \\
\hline
\end{tabular}

Connections significant at the threshold of $p<0.05$ corrected for 20 connections are in bold.

Because the entire model space for four modulatory effects on five interhemispheric connections is $4^{10}=1,048,576$, we used two procedures to reduce the number of models and simplify the analysis. (1) Because the main goal of the study was to examine reciprocality of interhemispheric connections in phonological processing with no specific hypothesis on different lexical conditions, all four lexical conditions were assumed to modulate the same connections within each model. (2) Our model selection procedure differed in two ways from standard approaches as used in the context of dynamic causal modeling. First, instead of testing all models within a single space of models, we used a hierarchical approach where we initially optimized the modulation of interhemispheric connections at the level of STG by using a set of eight models. The optimal model from this initial comparison was then chosen as a basis for creating a second set of models that varied with regard to the modulation of interhemispheric connections at the level of A1. While this approach does not guarantee that the same optimal model is found that would have been obtained had we tested for all possible combinations of modulatory influences on connections, it reduces the number of model comparisons by several orders of magnitude. Second, we used model space partitioning and tested three different partitions for the STG level models (or two different partitions for the A1 level models), each of which consisted of two model sets that differed with regard to the absence or presence of a particular modulatory influence. This corresponds to having a model space with a three-factorial structure, where each of the three interhemispheric modulatory influences constitutes one factor with two levels (i.e., absent or present).

All models shared the same intrinsic connections (see Table 3 ) and the bilinear effects on intrahemispheric connections (reciprocal connections between left IFG, FG, and STG and reciprocal connections between A1 and STG within each hemisphere) (Fig. 2). In all models, the auditory input (a regressor of all auditory stimuli, including words and pure tones) was specified as driving A1 bilaterally.

The goal of the first set of models was to determine the contribution of right STG to regions involved in phonological processing (i.e., left STG and IFG) and determine the reciprocality between left and right STGs. Modulation on three interhemispheric connections were tested: (1) left STG to right STG; (2) right STG to left STG; and (3) right STG to IFG; (Fig. 2). The goal of the second set of models was to determine the connectivity at the level of sensory auditory cortices, and it therefore manipulated the modulatory effect on right to left $\mathrm{A} 1$ and left to right $\mathrm{A} 1$ ) (Fig. 2). In cases where space partitioning did not reveal clear evidence in favor of including or rejecting a parameter, the more comprehensive model that included the parameter in question was selected to test the significance of these parameters and to avoid drawing strong conclusions from inconclusive results. Furthermore, because inconclusive results may arise from individual variability in the contribution of the relevant parameter and because previous studies suggest that there are individual differences in interhemispheric connectivity (e.g., sex, IQ, and age differences) (Schmithorst and Holland, 2007), selecting the model that includes the relevant parameter would enable a test of individual differences in this parameter.

\section{Statistical analysis of parameters within the selected model}

Second level analysis was performed on parameter estimates in the selected model using a random effects frequentist approach and Bonferroni correction for multiple comparisons. The significance of intrinsic connections and of the modulation of separate lexical conditions on each
A
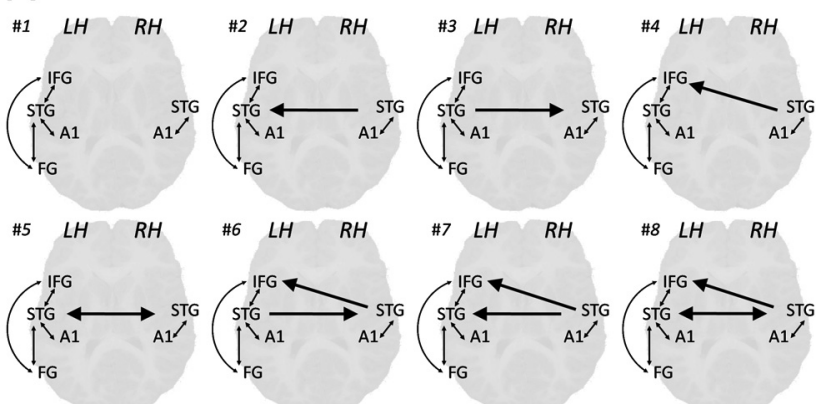

B
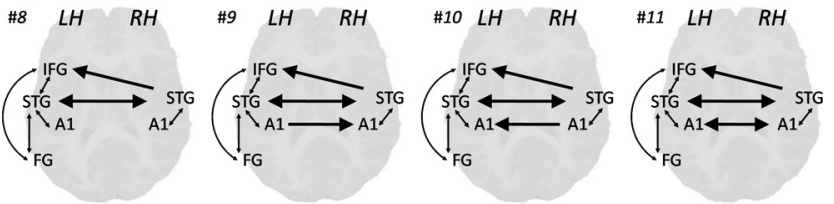

Figure 2. Modulatory effects in the alternative DCM models for model comparison. $A$, Models compared at the first stage, testing interhemispheric connectivity of right STG. $\boldsymbol{B}$, Models compared at the second stage, testing interhemispheric connectivity between left and right A1. Arrows indicate modulated connections, bold arrows indicate interhemispheric modulations. LH, Left hemisphere; $\mathrm{RH}$, right hemisphere.

connection was tested using one sample $t$ test $(p<0.05$, corrected for 20 connections for the intrinsic connections and for 14 connections for the modulatory effects). To determine the main direction of influence between hemispheres, two general linear model (GLM) analyses were conducted on the bilinear effects of connections between right and left STG and between right and left A1 separately. Each of these analyses included two directions and four conditions as within subject variables, gender as a between subject variable, with age, accuracy in the scanner, and VIQ scores as covariates. VIQ was included as a covariate to rule out the possibility that emerging gender effects were due to differences in language skills between the two gender groups. Finally, to determine hemispheric differences in intrahemispheric connectivity, we conducted a GLM analysis on bilinear effects on the connections between A1 and STG in the two hemispheres, with 2 hemispheres $\times 2$ directions $\times 4$ conditions as within-subject variables and gender as a between-subject variable.

\section{Results}

\section{Behavioral results}

Accuracy in four lexical conditions were entered into a GLM analysis, with four conditions as a within-subject variable, gender as a between-subject variable, and age and VIQ score as covariates. Significant effects of condition $\left(F_{(3,102)}=6.36, p<0.001\right)$ and gen$\operatorname{der}\left(F_{(1,34)}=9.33, p<0.01\right)$ were found. Figure $3 A$ shows that the condition with lowest accuracy was $\mathrm{O}^{+} \mathrm{P}^{-}$(e.g., pint-mint) and that girls were more accurate than boys across all conditions. A significant effect of age $\left(F_{(1,34)}=5.25, p<0.05\right)$ showed that accuracy in all conditions increased with age. A significant interaction of condition and VIQ $\left(F_{(3,105)}=3.27, p<0.05\right)$ was followed up by testing the correlation of accuracy and VIQ (controlled for age) separately in each condition. A significant increase in accuracy with VIQ was evident in the two nonrhyming conditions $\left(r=0.43, p<0.01\right.$ for the conflicting $\mathrm{O}^{+} \mathrm{P}^{-}$, and $r=0.32, p<0.05$ for the nonconflicting $\mathrm{O}^{-} \mathrm{P}^{-}$).

A similar GLM analysis was conducted for reaction time (RT) as a dependent variable and showed significant main effects of condition $\left(F_{(3,108)}=3.63, p<0.05\right)$, gender $\left(F_{(1,36)}=8.20, p<\right.$ $0.01)$, age $\left(F_{(1,34)}=7.36, p<0.05\right)$, and VIQ $\left(F_{(1,34)}=4.61, p<\right.$ $0.05)$. Figure $3 B$ shows that girls performed faster than boys 
A

1

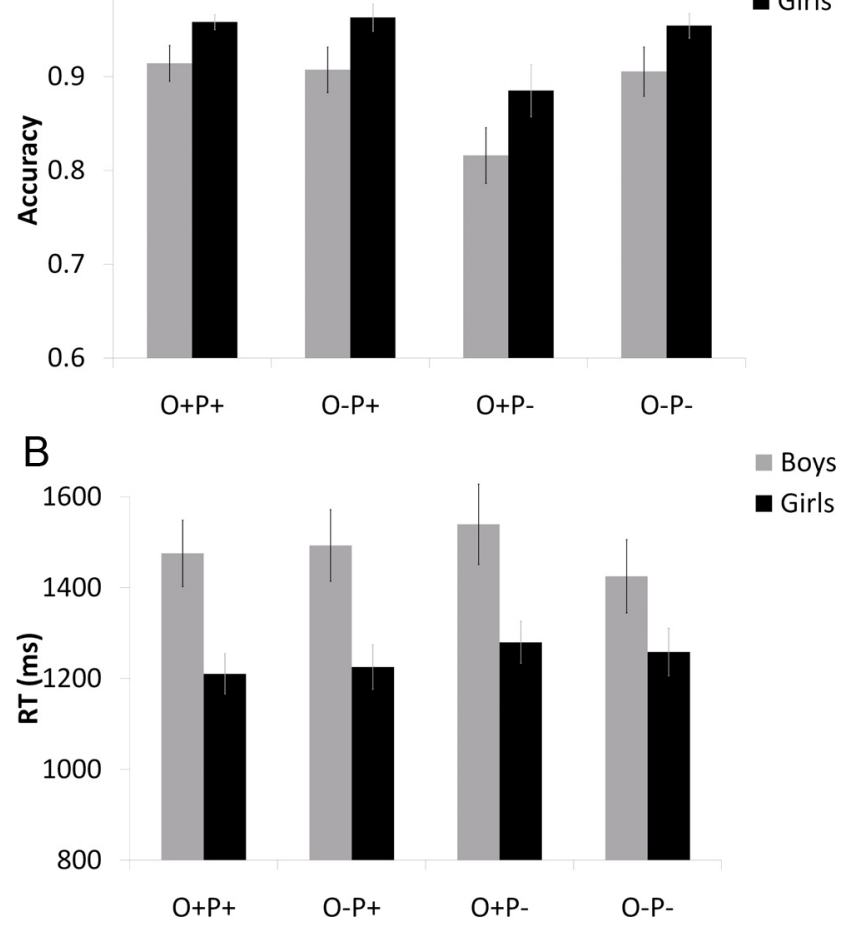

Figure 3. Performance in the scanner for girls (black) and boys (gray). $A$, Accuracy, $B$, Reaction time.

across all conditions. A significant interaction of condition and $\operatorname{VIQ}\left(F_{(3,102)}=2.85, p<0.05\right)$ was followed up by testing the correlation of RT with VIQ (controlled for age) separately in each condition. RT decreased with the increase in VIQ in the two nonrhyming conditions, with a significant correlation in the nonconflicting condition $\mathrm{O}^{-} \mathrm{P}^{-}(r=-0.40, p<0.01)$ and a nonsignificant trend in the conflicting condition $\mathrm{O}^{+} \mathrm{P}^{-}(r=$ $-0.23, p=0.087)$.

\section{Conventional analysis}

Table 2 and Figure 1 present regions that were active in the rhyming task for all lexical conditions compared to fixation. These include IFG and left FG, as well as bilateral superior temporal gyri (left and right STG). These regions were included in the DCM analysis. The comparison of the perceptual conditions versus fixation resulted in activation in bilateral Heschl gyri, among other regions. The peak of this activation in [-54 - 159] and [54 126 ] were included as input regions in the DCM (left and right A1).

\section{Model comparisons}

Model space partitioning with a random effects procedure (Stephan et al., 2009) was used in two steps to determine the model with the optimal balance between fit and complexity. The first step included models $1-8$, which differed in modulation on interhemispheric connections with right STG (see Fig. 2A). The family of models that include modulations on right to left STG (models 2, 5, 7, and 8) compared to models that do not include modulations on this connection (models $1,3,4$, and 6) resulted in exceedance probability (xp) of 0.64 versus 0.36 , favoring the inclusion of this parameter. Similarly, the comparison of the family of models that include modulation on the connection from right STG to left IFG (models 4, 6, 7, and 8) versus models that do not include modulation on this connection (models 1, 2, 3, and 5) resulted in exceedance probability of 0.67 versus 0.33 , favoring the inclusion of this parameter. Finally, the comparison of models that include modulations on the connection from left STG to right STG (models 3, 5, 6, and 8) versus models that do not include modulations on this connection (models 1, 2, 4, and 7) did not reveal a clear advantage for either family $(\mathrm{xp}=0.53$ vs 0.47$)$.

Model comparison has therefore yielded clear evidence in favor of including modulation on the connections from right to left STG and from right STG to left IFG, but yielded inconclusive evidence for modulation of the connection from left to right STG. Because this parameter is critical for the theoretical question of reciprocality, to avoid rejecting it based on inconclusive results that may arise from large individual variability we decided to test individual differences in this parameter. To do that, the selected model should include the relevant parameter so that its significance in different subgroups can be tested. Model 8, which includes modulations on all three interhemispheric connections with right STG, was selected as the basis for the second set of models. It should be noted, however, that this selection did not affect the results obtained for comparing other parameters in the second stage of analysis. This will be shown by presenting the results of model 7 in the relevant section.

The second set of models (8-11) systematically tested modulation on the connections between right and left A1. Family comparison between models that include modulations on the connection from left to right A1 (models 9 and 11) versus models that do not include modulations on this connection (models 8 and 10) resulted in exceedance probability of 0.07 versus 0.93 , favoring the rejection of this parameter. Family comparison between models that include modulations on the connection from right to left A1 (models 9 and 11) versus models that do not include modulation on this connection (models 8 and 10) did not reveal a clear advantage to any family ( $x p=0.46$ vs 0.54 ). Based on model comparisons, we therefore reject the modulation on the connection from left to right A1 but include modulation on the connection from right to left A1, which did not yield conclusive evidence, to test its effects of individual differences. Therefore, model 10 was selected for further analysis (see Fig. 2).

\section{Analysis within the selected model}

One sample $t$ test was conducted on intrinsic connections in model 10. Table 3 shows that connections going from all regions into A1 bilaterally were significant and negative (except for left A1 to right A1), and all other connections were significant and positive. One sample $t$ test was conducted on the modulatory effects in model 10 separately in each condition $(p<0.05$, corrected for 14 connections). Figure 4 shows that the bidirectional connections between left and right STG were significantly modulated by all four lexical conditions, the connection from right STG to left IFG was significantly modulated only by the two conflicting conditions, and the connection from right to left A1 was significantly modulated only by the conflicting rhyming condition $\mathrm{O}^{-} \mathrm{P}^{+}$(jazz-has). Further analyses were performed on bilinear effects in model 10 .

\section{Interhemispheric connectivity}

To determine the main direction of influence between hemispheres, the bilinear effects on connections between right and left STG were entered into a GLM analysis with two directions and four conditions as within-subject variables and sex as a betweensubject variable, with age, accuracy in the scanner, and VIQ scores as covariates. We found a significant effect of direction, 


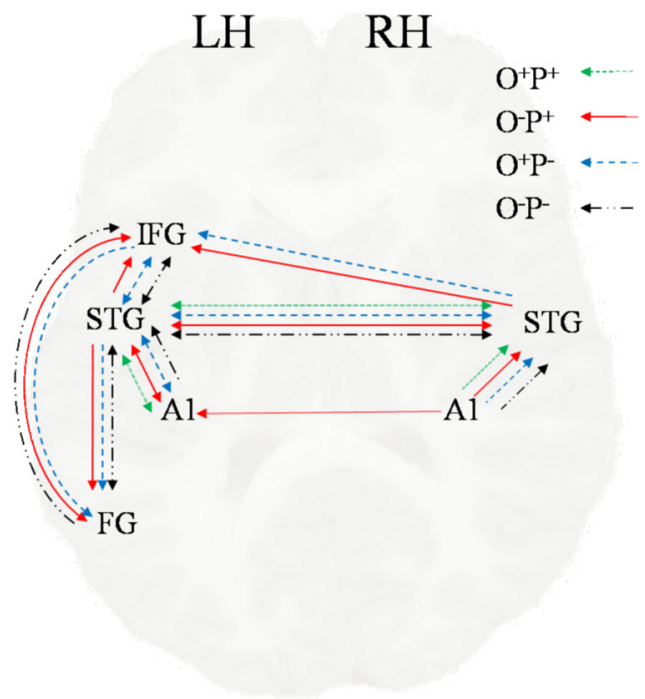

Figure 4. Significant modulations of the four lexical conditions on connections in model 10.

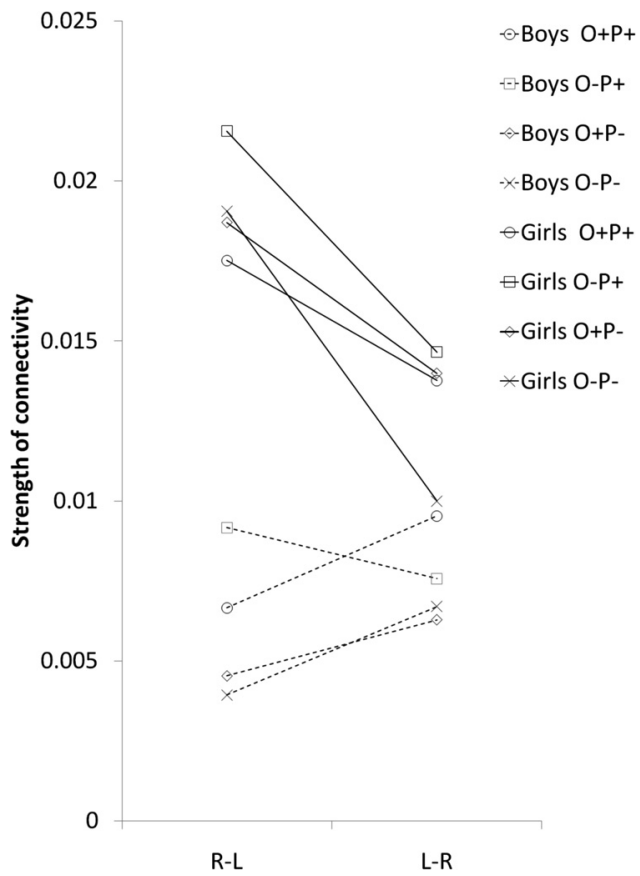

Figure 5. Differences between boys and girls in the modulation of interhemispheric connections between right and left STG. Modulations are presented separately for each condition in each direction. $R$, Right; $L$, left.

with stronger influence from right to left STG than from left to right STG $\left(F_{(1,34)}=11.26, p<0.01\right)$. However, there was also a significant effect of sex $\left(F_{(1,34)}=4.43, p<0.05\right)$, with stronger modulation in girls compared to boys and a significant interaction of sex and direction $\left(F_{(1,34)}=5.52, p<0.05\right)$. No main effect or interaction with age was found. Following the finding of this interaction, the effect of direction was tested separately within each sex in a GLM of direction and condition as within subject factors. In girls, modulation effects on the connection from right to left were significantly stronger than those on the connection from left to right $\left(F_{(1,18)}=5.92, p<0.05\right)$ (Fig. 5). Within boys, the pattern was significant in the opposite direction $\left(F_{(1,13)}=\right.$ 5.94, $p<0.05$ ), with stronger modulation of left to right compared with right to left connectivity. However, in boys there was a
A

Girls

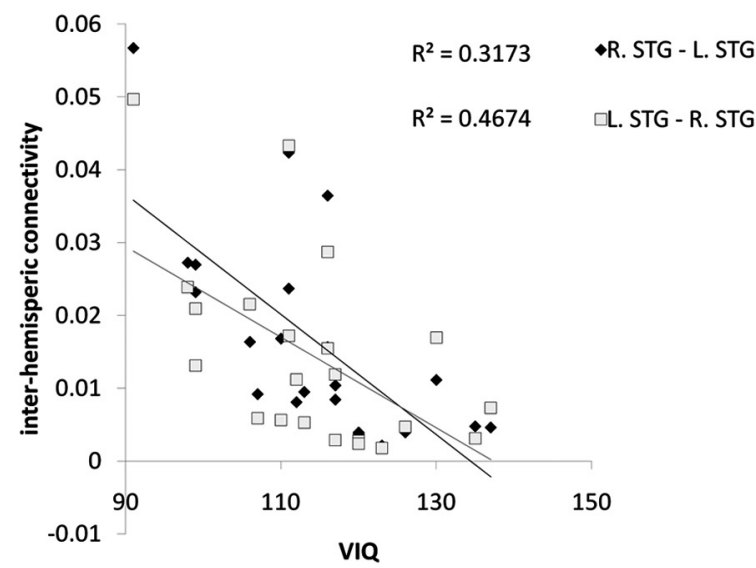

B

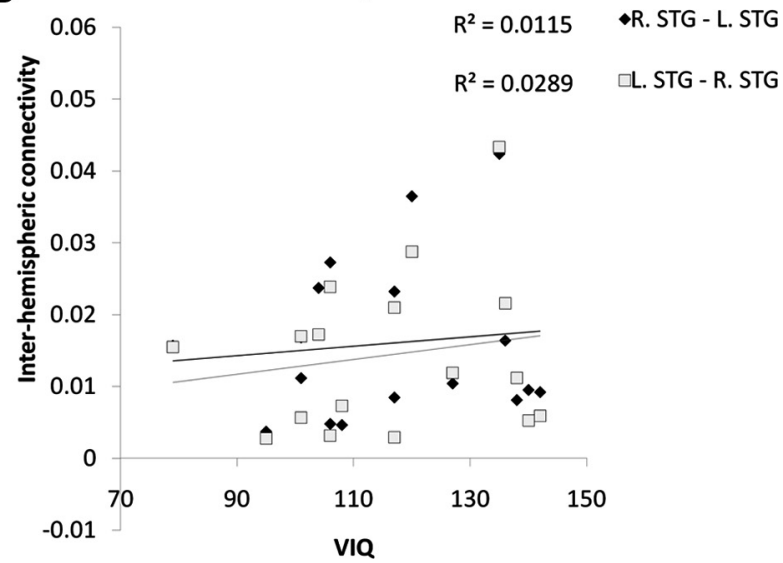

Figure 6. Correlation of VIQ with Inter-hemispheric connectivity between right and left STG, in girls $(\boldsymbol{A})$ and boys $(\boldsymbol{B})$. R, Right, $L$, left.

significant interaction of direction and condition $\left(F_{(3,39)}=3.76\right.$, $p<0.05$ ), because in the rhyming conflicting condition $\mathrm{O}^{-} \mathrm{P}^{+}$ (jazz-has), the effect of direction was similar to that of the girls (i.e., right to left $>$ left to right (Fig. 5).

Following the finding of significant sex differences in interhemispheric connectivity and because of existing differences in performance between boys and girls, the correlation between interhemispheric connectivity and performance measures, as well as measures of linguistic skill (verbal IQ), was tested separately for the two sexes. Within girls, a significant negative correlation was found between interhemispheric connectivity and VIQ scores (controlled for age and RT), as well as a positive correlation between interhemispheric connectivity and RT (controlled for age, and VIQ). Correlation coefficients with VIQ were $r=$ $-0.67), p<0.01$ for right STG-left STG and $r=-0.55), p<0.01$ for left STG-right STG across all conditions (Fig. 6). Correlation coefficients with RT were $r=0.40, p<0.05$ for right STG-left STG and $r=0.50, p<0.05$ for left STG-right STG across all conditions. In boys, no correlation was found between interhemispheric connectivity and RT or VIQ. No correlation was found between interhemispheric connectivity and accuracy in any of the sexes. These results raised the hypothesis that the sex difference in connectivity was only due to girls with low VIQ. To test this hypothesis, the entire sample was divided into low and high VIQ groups using a median split. A GLM analysis was conducted on the connections between right and left STG separately 
in each group with 2 directions $\times 4$ conditions as within subject variables, sex as a between subject factor, and age, accuracy, and VIQ as covariates. There was a significant effect of sex in the low VIQ group, with stronger connectivity in girls compared to boys $\left(F_{(1,14)}=5.264, p<0.05\right)$, but there was no effect of sex in the high VIQ group $\left(F_{(1,15)}<1\right)$.

A GLM analysis was conducted on the modulation of the connection from right $\mathrm{A} 1$ to left $\mathrm{A} 1$ to test for individual differences. Four conditions were entered as a within-subject variable, with sex as a between-subject variable and age, accuracy, and VIQ scores as covariates. A significant effect of sex was found $\left(F_{(1,34)}=\right.$ $5.25, p<0.05)$, with stronger connectivity in girls compared to boys. No effect of age was found, and no significant difference was found between conditions (despite the finding of the one sample $t$ test showing that only the rhyming conflicting condition $\mathrm{O}^{-} \mathrm{P}^{+}$(Jazz-Has) reached the significance threshold). A similar GLM analysis was conducted on the modulation of the connection from right STG to IFG, with no significant effects of sex or condition.

To test whether the effects of sex depends on the selection of model, the same analyses were done on the connection from right to left STG in model 7, and the results were similar: Modulations on this connection were significantly stronger for girls compared to boys $\left(F_{(1,34)}=6.8, p<0.05\right)$, and only girls showed a significant negative correlation with VIQ (controlled for RT and age; $r=-0.59, p<0.01)$. When participants were split into a high and low VIQ groups, only the low VIQ group showed a significant effect of $\operatorname{sex}\left(F_{(1,15)}=7.17, p<0.05\right)$ with no effect of sex in the high VIQ group $\left(F_{(1,16)}=2.26, p=0.15\right)$.

\section{Hemispheric differences in intrahemispheric connectivity}

We tested for asymmetry in connectivity within each hemisphere, by conducting a GLM analysis on the modulation of connections between A1 and STG. The analysis included 2 hemispheres $\times 2$ directions $\times 4$ conditions as within-subject variables and sex as a between-subject variable. The results showed a significant effect of hemisphere $\left(F_{(1,37)}=10.43, p<0.01\right)$, a significant effect of direction $\left(F_{(1,37)}=111.9, p<0.001\right)$, and a significant interaction of hemisphere and direction $\left(F_{(1,37)}=11.24, p<0.01\right)$, with stronger modulation within the left hemisphere, but only for forward connections. No effect of sex or age was found.

To test whether the asymmetry of intrahemispheric connectivity depends on the selection of model, the same analyses were done in model 7 and the results were similar: There was a significant interaction of direction $\times$ hemisphere $\left(F_{(1,37)}=9.78, p<\right.$ 0.01 ), showing that the difference between hemispheres is only evident for the forward connection.

\section{Discussion}

Our results showed reciprocal interactions between bilateral STG, and right to left connectivity at the sensory level (A1) in one condition. The influence of right STG on left IFG was significant in the conflicting conditions. Interhemispheric connectivity for girls was stronger compared with that for boys and stronger from right to left STG, compared to the opposite direction. Finally, despite girls' advantage in task performance, interhemispheric connectivity between bilateral STGs was associated with slow performance and low verbal IQ in girls. No correlation was found between age and interhemispheric connectivity.

\section{Reciprocality and levels of processing}

Reciprocal interactions between right and left STGs indicate hemispheric cooperation during the phonological task, consis- tent with synchronization between hemispheres in language tasks found in electrophysiological studies (Weiss and Mueller, 2003). Reciprocality may reflect integration of different sound processing outputs typical of the two hemispheres. It has been suggested that while left hemisphere A1 is sensitive to temporal information (Zatorre et al., 2002; Boemio et al., 2005), A1 on the right shows greater sensitivity for pitch modulations (Zatorre, 1988; Johnsrude et al., 2000; Lattner et al., 2005). Furthermore, left STG was depicted in tasks of phonological access (Scott et al., 2000; Booth et al., 2002; Bitan et al., 2007), while right STG was shown to be sensitive to speaker voice information (von Kriegstein et al., 2003; Lattner et al., 2005) and involved in processing intonation and prosody (Meyer et al., 2004; Ethofer et al., 2007; Wiethoff et al., 2008). However, other studies suggest that phonological level processing is mediated by both left and right temporal cortices, with only a mild leftward bias (Okada and Hickok, 2006; Hickok and Poeppel, 2007). According to the "asymmetric sampling in time" hypothesis (Poeppel, 2003), the left hemisphere preferentially extracts information from short temporal integration windows (namely segment-level representations), while the right hemisphere preferentially extracts information from long integration windows (namely, syllable-level representations). This can account for the phonological capabilities of the right hemisphere found in rhyming judgment tasks (Rayman and Zaidel, 1991). The rhyming task used in the current study involves determining whether suprasegmental information is similar across two items. Thus, the contribution of the right STG in the current study may reflect processing at the syllablelevel representations or processing information about the speaker's voice and intonation. In both cases, reciprocal connectivity between left and right STGs may reflect the integration of information from short and long temporal windows necessary for speech recognition.

Interhemispheric interactions were found at multiple levels of processing: between A1 cortices, between bilateral STG, and from right STG to left IFG, consistent with results from dichotic listening, electrophysiological, and animal studies (Pollmann et al., 2002; Aboitiz and Montiel, 2003; Weiss and Mueller, 2003). The left IFG was shown to be involved in a very wide range of cognitive processes, including phonological segmentation (Hagoort et al., 1999; Burton, 2009) relevant for rhyming judgments and integration and control of linguistic processes (Bitan et al., 2005; Hagoort, 2005). The direct influence of right STG on left IFG in the conflicting conditions is consistent with the notion of greater contribution of the nonspecialized hemisphere with increasing task demands (Banich, 1998; Weissman et al., 2000) and suggests that under conflicting conditions, sound processing information, carried out by right STG, contributes more to rhyming judgment.

One functional magnetic resonance imaging (MRI) study applied DCM analysis to examine interhemispheric connectivity by using letter judgment and spatial decision tasks in fMRI with lateralized visual presentation (Stephan et al., 2007). In the letter judgment task they found right to left influence in lingual gyrus only with right hemisphere presentation. However, in the spatial task bidirectional influences were found between the superior parietal cortices independent of the side of presentation, suggesting that the level and direction of interhemispheric interactions depend on the task. Compared with the letter judgment task, the current study differs not only in the input modality, age of participants, and bilateral presentation but also in the complexity of the task, all of which may have contributed to increased interhemispheric connectivity at higher levels of processing and to increased reciprocality. 


\section{Asymmetry}

Despite the reciprocal interaction, there is evidence for hemispheric asymmetry, consistent with left hemisphere specialization for phonological processing. Intrahemispheric connections from A1 to STG were stronger in the left hemisphere, consistent with findings of correlation between structural or functional connectivity in the left hemisphere and language lateralization (Klingberg et al., 2000; Gold et al., 2006). Interhemispheric connectivity was stronger from right to left at the level of A1 (unidirectional influence) and at the level of STG in girls. Evoked potential studies with lateralized presentation showed faster transmission from the nonspecialized to the specialized hemisphere (Nowicka et al., 1996; Krumbholz et al., 2007) consistent with the current results. In contrast, a positron emission tomography study that used structural equation modeling in face and location matching tasks, found stronger connections from the specialized to the nonspecialized hemisphere (McIntosh et al., 1994). Influences in this direction may represent signals from the specialized hemisphere for recruiting the nonspecialized hemisphere (Weissman et al., 2000; Stephan et al., 2007).

Altogether, the results are consistent with the specialization of the left hemisphere for phonological processing. The influence of the right STG may contribute voice spectral information or syllable level phonological information, which is integrated into the phonological (segmental) information represented in left STG. The right STG may also directly affect higher level processing in the left hemisphere in dorsal IFG (pars opercularis and pars triangularis).

\section{Sex differences}

We found stronger interhemispheric connectivity for girls compared to boys (in A1 and STG). However, despite the girls' advantage in task performance, interhemispheric connectivity in girls was associated with slower performance and with low verbal IQ. These results suggest that excessive interhemispheric connectivity may sometimes interfere with performance, at least in a phonological decision task.

Early studies hypothesized that females' bilateral language representation explains their superior verbal skills (Levy, 1969). Supporting evidence included anatomical and structural imaging studies showing less hemispheric asymmetry of the planum temporale (Wada et al., 1975; Foundas et al., 2002) and larger corpus callosum in females compared to males (Steinmetz et al., 1992; Bamiou et al., 2007). Moreover, the volume of the corpus callosum was positively correlated with cognitive performance in females (Davatzikos and Resnick, 1998; Luders et al., 2007). Nevertheless, other studies failed to find sex differences in structural hemispheric asymmetry or in the size of the corpus callosum (Aboitiz et al., 1992; Jancke et al., 1994; Bishop and Wahlsten, 1997; Lebel and Beaulieu, 2009). Several functional imaging studies showed sex differences in lateralization in adults with more bilateral activation in females compared to males (Shaywitz et al., 1995; Jaeger et al., 1998; Kansaku and Kitazawa, 2001; Phillips et al., 2001). However, other functional imaging studies did not find any sex differences in language lateralization (Frost et al., 1999; Sommer et al., 2004), revealing the heated controversy in the literature.

Previous studies show that sex differences in language lateralization are task dependent (Sommer et al., 2004; Kitazawa and Kansaku, 2005) and limited to specific regions (Kansaku et al., 2000) and timing parameters (Ortigue et al., 2005). The dynamic nature of language lateralization may explain why sex differences are not always evident in static measures of structural interhemi- spheric connectivity and in measures of local functional activation. Effective connectivity analysis, as a measure of dynamic changes to hemispheric communication, may thus be more sensitive to sex-related differences in hemispheric interactions. One previous fMRI study found sex differences in interhemispheric functional connectivity (Schmithorst and Holland, 2007). In this study, which used a narrative comprehension task in children, interhemispheric connectivity among temporal and frontal language areas was positively correlated with IQ in both sexes. However, the specific connections that showed this correlation were different for each sex. Despite its support for sex differences in interhemispheric communication, these results are inconsistent with the current study, which found a negative correlation between interhemispheric connectivity and VIQ in girls. In addition to the use of different IQ measures in the two studies, a potential explanation for this discrepancy is the different tasks employed in the studies. Narrative comprehension may benefit more from interhemispheric interactions because it relies more heavily on the integration of sentence prosody and syntactic information (Friederici et al., 2007). In contrast, for the phonological decision required for the rhyming task, too much reliance on melodic pitch patterns and the speaker's voice information contributed by the right STG may interfere with performance. Thus, strong cooperation between hemispheres may not always contribute to efficient cognitive processing (Golestani et al., 2007; Everts et al., 2009; Lebel and Beaulieu, 2009). If the female brain has the structural potential for enhanced interhemispheric communication, it may prove beneficial in certain conditions, yet girls with low verbal skills may be ineffective in using it according to task requirements.

In contrast to our hypothesis and to previous studies that showed a developmental increase in language lateralization (Boles et al., 2008; Brauer et al., 2008; Ressel et al., 2008; Everts et al., 2009), there was no effect of age on interhemispheric connectivity. This may be due to the smaller range of ages used in the current study as compared to the above studies. We also did not find any interaction between age and sex. However, findings from the narrative comprehension task that found increasing sex differences with age (Schmithorst and Holland, 2007) suggest that sex differences found for children in the current study may also be true for adults.

In conclusion, our results suggest that during phonological processing reciprocal interhemispheric interactions occur at multiple levels of processing, showing that outcomes from right hemisphere processing are integrated into the final phonological decisions in the left hemisphere. Our findings show more interhemispheric connectivity for girls compared to boys, but this seems to interfere with task performance. We suggest that girls may have the potential for more interhemispheric communication, which may be beneficial in some tasks, but girls with low verbal skills may be ineffective in modulating interhemispheric connectivity according to task requirements.

\section{References}

Aboitiz F, Montiel J (2003) One hundred million years of interhemispheric communication: the history of the corpus callosum. Braz J Med Biol Res 36:409-420.

Aboitiz F, Scheibel AB, Zaidel E (1992) Morphometry of the sylvian fissure and the corpus-callosum, with emphasis on sex-differences. Brain 115:1521-1541.

Bamiou DE, Sisodiya S, Musiek FE, Luxon LM (2007) The role of the interhemispheric pathway in hearing. Brain Res Rev 56:170-182.

Banich MT (1998) The missing link: the role of interhemispheric interaction in attentional processing. Brain Cogn 36:128-157. 
Baayan RH, Piepenbrock R, Gulikers L (1995) The CELEX lexical database (CD-ROM). Philadelphia: Linguistic Data Consortium, University of Pennsylvania.

Binder JR, Rao SM, Hammeke TA, Yetkin FZ, Jesmanowicz A, Bandettini PA, Wong EC, Estkowski LD, Goldstein MD, Haughton VM, Hyde JS (1994) Functional magnetic resonance imaging of human auditory cortex. Ann Neurol 35:662-672.

Bishop KM, Wahlsten D (1997) Sex differences in the human corpus callosum: myth or reality? Neurosci Biobehav Rev 21:581-601.

Bitan T, Booth JR, Choy J, Burman DD, Gitelman DR, Mesulam MM (2005) Shifts of effective connectivity within a language network during rhyming and spelling. J Neurosci 25:5397-5403.

Bitan T, Burman DD, Chou TL, Lu D, Cone NE, Cao F, Bigio JD, Booth JR (2007) The interaction between orthographic and phonological information in children: an fMRI study. Hum Brain Mapp 28:880-891.

Bloom JS, Hynd GW (2005) The role of the corpus callosum in interhemispheric transfer of information: excitation or inhibition? Neuropsychol Rev 15:59-71.

Boemio A, Fromm S, Braun A, Poeppel D (2005) Hierarchical and asymmetric temporal sensitivity in human auditory cortices. Nat Neurosci 8:389-395.

Bogen JE (2000) Split-brain basics: relevance for the concept of one's other mind. J Am Acad Psychoanal 28:341-369.

Boles DB, Barth JM, Merrill EC (2008) Asymmetry and performance: toward a neurodevelopmental theory. Brain Cogn 66:124-139.

Booth JR, Burman DD, Meyer JR, Gitelman DR, Parrish TB, Mesulam MM (2002) Modality independence of word comprehension. Hum Brain Mapp 16:251-261.

Booth JR, Burman DD, Meyer JR, Gitelman DR, Parrish TB, Mesulam MM (2004) Development of brain mechanisms for processing orthographic and phonologic representations. J Cogn Neurosci 16:1234-1249.

Brauer J, Neumann J, Friederici AD (2008) Temporal dynamics of perisylvian activation during language processing in children and adults. Neuroimage 41:1484-1492.

Britton B, Blumstein SE, Myers EB, Grindrod C (2009) The role of spectral and durational properties on hemispheric asymmetries in vowel perception. Neuropsychologia 47:1096-1106.

Burman DD, Bitan T, Booth JR (2008) Sex differences in neural processing of language among children. Neuropsychologia 46:1349-1362.

Burock MA, Buckner RL, Woldorff MG, Rosen BR, Dale AM (1998) Randomized event-related experimental designs allow for extremely rapid presentation rates using functional MRI. Neuroreport 16:3735-3739.

Burton MW (2009) Understanding the role of the prefrontal cortex in phonological processing. Clin Linguist Phon 23:180-195.

Cone NE, Burman DD, Bitan T, Bolger DJ, Booth JR (2008) Developmental changes in brain regions involved in phonological and orthographic processing during spoken language processing. Neuroimage 41:623-635.

Davatzikos C, Resnick SM (1998) Sex differences in anatomic measures of interhemispheric connectivity: correlations with cognition in women but not men. Cereb Cortex 8:635-640.

Endrass T, Mohr B, Rockstroh B (2002) Reduced interhemispheric transmission in schizophrenia patients: evidence from event-related potentials. Neurosci Lett 320:57-60.

Ethofer T, Wiethoff S, Anders S, Kreifelts B, Grodd W, Wildgruber D (2007) The voices of seduction: cross-gender effects in processing of erotic prosody. Soc Cogn Affect Neurosci 2:334-337.

Everts R, Lidzba K, Wilke M, Kiefer C, Mordasini M, Schroth G, Perrig W, Steinlin M (2009) Strengthening of laterality of verbal and visuospatial functions during childhood and adolescence. Hum Brain Mapp 30:473-483.

Foundas AL, Leonard CM, Hanna-Pladdy B (2002) Variability in the anatomy of the planum temporale and posterior ascending ramus: do rightand left-handers differ? Brain Lang 83:403-424.

Friederici AD, von Cramon DY, Kotz SA (2007) Role of the corpus callosum in speech comprehension: interfacing syntax and prosody. Neuron 53:135-145.

Frost JA, Binder JR, Springer JA, Hammeke TA, Bellgowan PS, Rao SM, Cox RW (1999) Language processing is strongly left lateralized in both sexes: evidence from functional MRI. Brain 122:199-208.

Galaburda AM, Rosen GD, Sherman GF (1990) Individual variability in cortical organization: Its relationship to brain laterality and implications to function. Neuropsychologia 28:529-546.
Gazzaniga MS (2000) Cerebral specialization and interhemispheric communication: does the corpus callosum enable the human condition? Brain 123:1293-1326.

Gold BT, Balota DA, Jones SJ, Powell DK, Smith CD, Andersen AH (2006) Dissociation of automatic and strategic lexical-semantics: functional magnetic resonance imaging evidence for differing roles of multiple frontotemporal regions. J Neurosci 26:6523-6532.

Golestani N, Molko N, Dehaene S, LeBihan D, Pallier C (2007) Brain structure predicts the learning of foreign speech sounds. Cereb Cortex 17:575-582.

Grimshaw GM, Kwasny KM, Covell E, Johnson RA (2003) The dynamic nature of language lateralization: effects of lexical and prosodic factors. Neuropsychologia 41:1008-1019.

Hagoort P (2005) On Broca, brain, and binding: a new framework. Trends Cogn Sci 9:416-423.

Hagoort P, Indefrey P, Brown C, Herzog H, Steinmetz H, Seitz RJ (1999) The neural circuitry involved in the reading of German words and pseudowords: a PET study. J Cogn Neurosci 11:383-398.

Hellige JB (1990) Hemispheric asymmetry. Annu Rev Psychol 41:55-80.

Henson R, Buchel C, Josephs O, Friston KJ (1999) The slice-timing problem in event-related fMRI. Neuroimage [Suppl] 9:S125.

Hickok G, Poeppel D (2007) Opinion - The cortical organization of speech processing. Nat Rev Neurosci 8:393-402.

Hugdahl K, Carlsson G, Uvebrant P, Lundervold AJ (1997) Dichoticlistening performance and intracarotid injections of amobarbital in children and adolescents: preoperative and postoperative comparisons. Arch Neurol 54:1494-1500.

Iacoboni M, Zaidel E (1996) Hemispheric independence in word recognition: evidence from unilateral and bilateral presentations. Brain Lang 53:121-140.

Jaeger JJ, Lockwood AH, Van Valin RD Jr, Kemmerer DL, Murphy BW, Wack DS (1998) Sex differences in brain regions activated by grammatical and reading tasks. Neuroreport 9:2803-2807.

Jäncke L, Schlaug G, Huang Y, Steinmetz H (1994) Asymmetry of the planum parietale. Neuroreport 5:1161-1163.

Jansen A, Menke R, Sommer J, Förster AF, Bruchmann S, Hempleman J, Weber B, Knecht S (2006) The assessment of hemispheric lateralization in functional MRI - Robustness and reproducibility. Neuroimage 33:204-217.

Johnsrude IS, Penhune VB, Zatorre RJ (2000) Functional specificity in the right human auditory cortex for perceiving pitch direction. Brain 123:155-163.

Kansaku K, Kitazawa S (2001) Imaging studies on sex differences in the lateralization of language. Neurosci Res 41:333-337.

Kansaku K, Yamaura A, Kitazawa S (2000) Sex differences in lateralization revealed in the posterior language areas. Cereb Cortex 10:866-872.

Kimura D (1961) Some effects of temporal-lobe damage on auditory perception. Can J Psychol 15:156-165.

Kinsbourne M (1982) Hemispheric-specialization and the growth of human understanding. Am Psychol 37:411-420.

Kitazawa S, Kansaku K (2005) Sex difference in language lateralization may be task-dependent. Brain 128:E30; author reply E31.

Klingberg T, Hedehus M, Temple E, Salz T, Gabrieli JD, Moseley ME, Poldrack RA (2000) Microstructure of temporo-parietal white matter as a basis for reading ability: evidence from diffusion tensor magnetic resonance imaging. Neuron 25:493-500.

Krumbholz K, Hewson-Stoate N, Schönwiesner M (2007) Cortical response to auditory motion suggests an asymmetry in the reliance on interhemispheric connections between the left and right auditory cortices. J Neurophysiol 97:1649-1655.

Lattner S, Meyer ME, Friederici AD (2005) Voice perception: Sex, pitch, and the right hemisphere. Hum Brain Mapp 24:11-20.

Lebel C, Beaulieu C (2009) Lateralization of the arcuate fasciculus from childhood to adulthood and its relation to cognitive abilities in children. Hum Brain Mapp 30:3563-3573.

Levy J (1969) Possible basis for evolution of lateral specialization of human brain. Nature 224:614-615.

Luders E, Narr KL, Bilder RM, Thompson PM, Szeszko PR, Hamilton L, Toga AW (2007) Positive correlations between corpus callosum thickness and intelligence. Neuroimage 37:1457-1464.

Maldjian JA, Laurienti PJ, Kraft RA, Burdette JH (2003) An automated 
method for neuroanatomic and cytoarchitectonic atlas-based interrogation of fMRI data sets. Neuroimage 19:1233-1239.

McIntosh AR, Grady CL, Ungerleider LG, Haxby JV, Rapoport SI, Horwitz B (1994) Network analysis of cortical visual pathways mapped with PET. J Neurosci 14:655-666.

Mechelli A, Price CJ, Noppeney U, Friston KJ (2003) A dynamic causal modeling study on category effects: bottom-up or top-down mediation? J Cogn Neurosci 15:925-934.

Meyer M, Steinhauer K, Alter K, Friederici AD, von Cramon DY (2004) Brain activity varies with modulation of dynamic pitch variance in sentence melody. Brain Lang 89:277-289.

Mohr B, Pulvermüller F, Rayman J, Zaidel E (1994) Interhemispheric cooperation during lexical processing is mediated by the corpus callosum: evidence from the split-brain. Neurosci Lett 181:17-21.

Musiek FE, Kurdziel-Schwan S, Kibbe KS, Gollegly KM, Baran JA, Rintelmann WF (1989) The dichotic rhyme task: results in split-brain patients. Ear Hear 10:33-39.

Nowicka A, Grabowska A, Fersten E (1996) Interhemispheric transmission of information and functional asymmetry of the human brain. Neuropsychologia 34:147-151.

Okada K, Hickok G (2006) Identification of lexical-phonological networks in the superior temporal sulcus using functional magnetic resonance imaging. Neuroreport 17:1293-1296.

Ortigue S, Thut G, Landis T, Michel CM (2005) Time-resolved sex differences in language lateralization. Brain 128:E28; author reply E29.

Penny WD, Stephan KE, Mechelli A, Friston KJ (2004) Comparing dynamic causal models. Neuroimage 22:1157-1172.

Petit L, Simon G, Joliot M, Andersson F, Bertin T, Zago L, Mellet E, TzourioMazoyer N (2007) Right hemisphere dominance for auditory attention and its modulation by eye position: an event related fMRI study. Restor Neurol Neurosci 25:211-225.

Phillips MD, Lowe MJ, Lurito JT, Dzemidzic M, Mathews VP (2001) Temporal lobe activation demonstrates sex-based differences during passive listening. Radiology 220:202-207.

Poeppel D (2003) The analysis of speech in different temporal integration windows: cerebral lateralization as 'asymmetric sampling in time.' Speech Commun 41:245-255.

Pollmann S, Maertens M, von Cramon DY, Lepsien J, Hugdahl K (2002) Dichotic listening in patients with splenial and nonsplenial callosal lesions. Neuropsychology 16:56-64.

Rayman J, Zaidel E (1991) Rhyming and the right hemisphere. Brain Lang 40:89-105.

Ressel V, Wilke M, Lidzba K, Lutzenberger W, Krägeloh-Mann I (2008) Increases in language lateralization in normal children as observed using magnetoencephalography. Brain Lang 106:167-176.

Schmithorst VJ, Holland SK (2007) Sex differences in the development of neuroanatomical functional connectivity underlying intelligence found using Bayesian connectivity analysis. Neuroimage 35:406-419.

Scott SK, Blank CC, Rosen S, Wise RJ (2000) Identification of a pathway for intelligible speech in the left temporal lobe. Brain 123:2400-2406.
Shaywitz BA, Shaywitz SE, Pugh KR, Constable RT, Skudlarski P, Fulbright RK, Bronen RA, Fletcher JM, Shankweiler DP, Katz L, Gore JC (1995) Sex-differences in the functional-organization of the brain for language. Nature 373:607-609.

Sommer IE, Aleman A, Bouma A, Kahn RS (2004) Do women really have more bilateral language representation than men? A meta-analysis of functional imaging studies. Brain 127:1845-1852.

Steinmetz H, Jäncke L, Kleinschmidt A, Schlaug G, Volkmann J, Huang Y (1992) Sex but no hand difference in the isthmus of the corpus-callosum. Neurology 42:749-752.

Stephan KE, Marshall JC, Penny WD, Friston KJ, Fink GR (2007) Interhemispheric integration of visual processing during task-driven lateralization. J Neurosci 27:3512-3522.

Stephan KE, Penny WD, Daunizeau J, Moran RJ, Friston KJ (2009) Bayesian model selection for group studies. Neuroimage 46:1004-1017.

Sugishita M, Otomo K, Yamazaki K, Shimizu H, Yoshioka M, Shinohara A (1995) Dichotic-listening in patients with partial section of the corpuscallosum. Brain 118:417-427.

von Kriegstein K, Eger E, Kleinschmidt A, Giraud AL (2003) Modulation of neural responses to speech by directing attention to voices or verbal content. Brain Res Cogn Brain Res 17:48-55.

Wada JA, Clarke R, Hamm A (1975) Cerebral hemispheric asymmetry in humans: cortical speech zones in 100 adult and 100 infant brains. Arch Neurol 32:239-246.

Wechsler D (1999) Wechsler abbreviated scale of intelligence. San Antonio, TX: The Psychological Corporation.

Weems SA, Reggia JA (2004) Hemispheric specialization and independence for word recognition: a comparison of three computational models. Brain Lang 89:554-568.

Weiss S, Mueller HM (2003) The contribution of EEG coherence to the investigation of language. Brain Lang 85:325-343.

Weissman DH, Banich MT, Puente EI (2000) An unbalanced distribution of inputs across the hemispheres facilitates interhemispheric interaction. J Int Neuropsychol Soc 6:313-321.

Westerhausen R, Woerner W, Kreuder F, Schweiger E, Hugdahl K, Wittling W (2006) The role of the corpus callosum in dichotic listening: a combined morphological and diffusion tensor imaging study. Neuropsychology 20:272-279.

Wiethoff S, Wildgruber D, Kreifelts B, Becker H, Herbert C, Grodd W, Ethofer T (2008) Cerebral processing of emotional prosody: influence of acoustic parameters and arousal. Neuroimage 39:885-893.

Zatorre RJ (1988) Pitch perception of complex tones and human temporallobe function. J Acoust Soc Am 84:566-572.

Zatorre RJ, Belin P, Penhune VB (2002) Structure and function of auditory cortex: music and speech. Trends Cogn Sci 6:37-46.

Zeno SM, Ivens SH, Millard RT, Duvvuri R (1996) The educator's word frequency guide (CD-ROM, DOS version). Brewster, NY: Touchstone Applied Science Associates. 